\title{
LA-UR-11- O6:0.5i
}

Approved for public release:

distribution is unlimited.

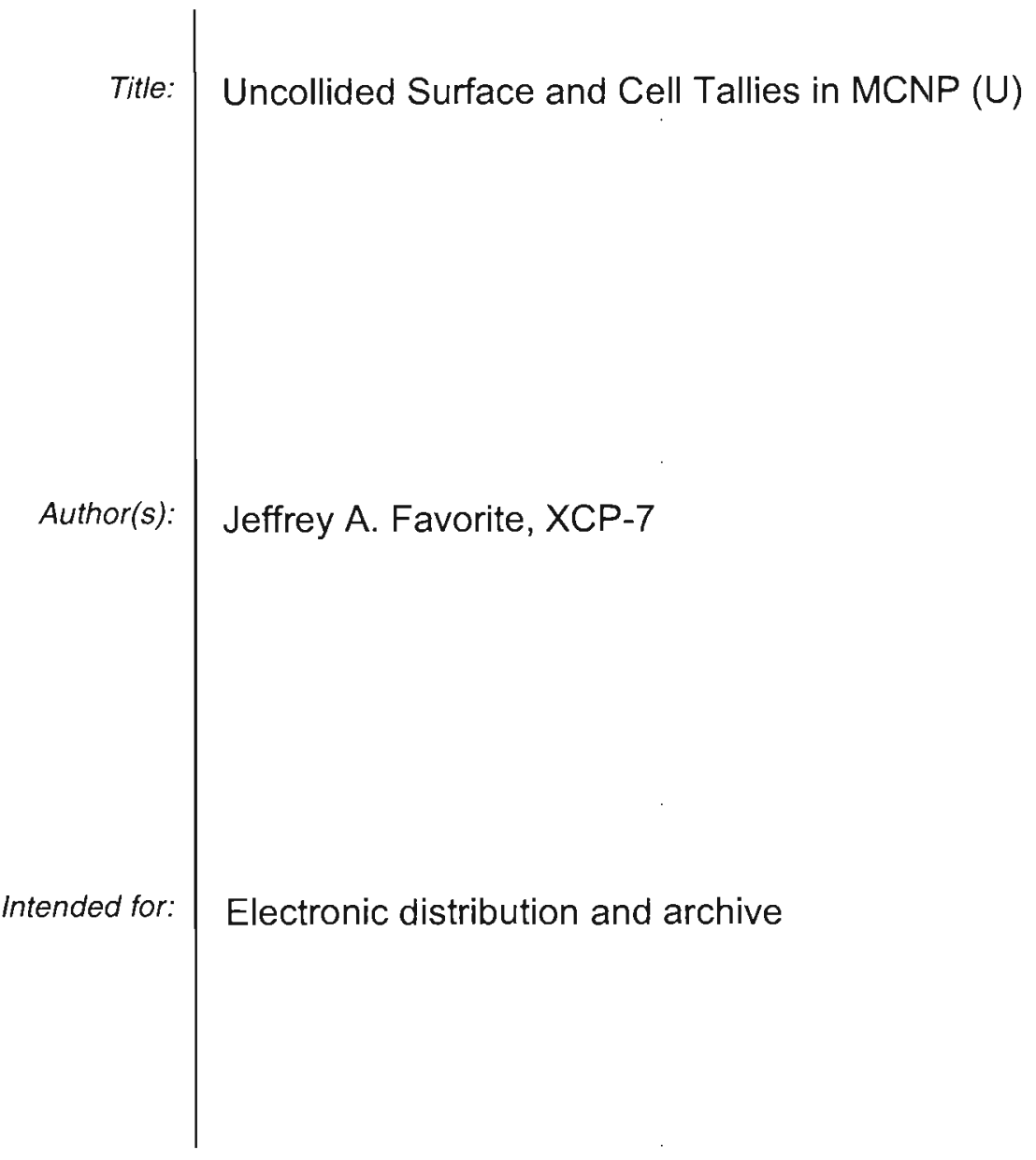

\section{- LOSA Alamos} EST. 1943

Los Alamos National Laboratory, an affirmative action/equal opportunity employer, is operated by the Los Alamos National Security, LLC for the National Nuclear Security Administration of the U.S. Department of Energy under contract DE-AC52-06NA25396. By acceptance of this article, the publisher recognizes that the U.S. Government retains a nonexclusive, royalty-free license to publish or reproduce the published form of this contribution, or to allow others to do so, for U.S. Government purposes: Los Alamos National Laboratory requests that the publisher identify this article as work performed under the auspices of the U.S. Department of Energy. Los Alamos National Laboratory strongly supports academic freedom and a researcher's right to publish; as an institution, however, the Laboratory does not endorse the viewpoint of a publication or guarantee its technical correctness. 


\title{
Los Alamos
}

NATIONAL LABORATORY

research note

\section{X-Computational Physics Division}

Transport Applications Group

Group XCP-7, MS F699

Los Alamos, New Mexico 87545

505/606-0495

Fax: $505 / 667-3726$

\author{
To/MS: Distribution \\ From/MS: Jeffrey A. Favorite / XCP-7, MS F663 \\ Phone/Email: 7-7941 / fave@lanl.gov \\ Symbol: XCP-7-RN(U)12-01 (LA-UR-11-????) \\ Date: October 20,2011
}

\section{SUBJECT: Uncollided Surface and Cell Tallies in MCNP (U)}

\begin{abstract}
Ray analysis can be very useful when the uncollided or "direct" contribution of a neutral source to a response of interest is desired. MCNP provides a ray analysis capability through the "no transport" or NOTRN feature, which calculates only uncollided contributions (direct from the source) to point-detector tallies. In this paper, the NOTRN capability is extended to provide uncollided contributions to surface and cell tallies. The user interface is unchanged; brief changes to the MCNP documentation are presented. Two verification problems are presented (one is analytic; the other is a comparison with PARTISN) and the capability is shown to be more efficient than the special tally treatment that tallies by the number of collisions. Six subroutines were modified.
\end{abstract}

\section{Introduction}

Ray analysis can be very powerful and useful when the uncollided or "direct" contribution of a neutral source to a response of interest is desired. "The MCNP Monte Carlo code ${ }^{2}$ provides a ray analysis capability through the "no transport" or NOTRN feature, which calculates only uncollided contributions (direct from the source) to point-detector tallies.

When the NOTRN capability is invoked, no surface or cell tallies are made. This need not be the case. It is also useful to compute the uncollided contributions to surface and cell tallies. ${ }^{3,4}$ In this paper, we document the source code modifications that were implemented to allow MCNP to compute uncollided surface and cell tallies quite easily in the

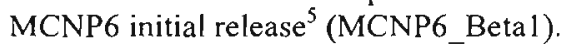

It is possible to obtain uncollided surface and cell tallies in MCNP using a special tally treatment to tally by the number of collisions. It is demonstrated in this paper that the NOTRN capability can provide uncollided results much more efficiently.

The theory and method are presented in the next section. Changes in the NOTRN user interface and the MCNP documentation are presented in Sec. III. Two verification problems are presented in Sec. IV. The source code modifications are detailed in Sec. V.

\section{Theory and Method}

Let the particle source density at a point in space be denoted $q(r, \hat{\mathbf{\Omega}})$. The cell containing the source is a homogenous material with macroscopic cross section $\Sigma$. The uncollided particle density along a ray in direction $\hat{\boldsymbol{\Omega}}$ a distance $s$ from the source point (i.e., the uncollided density that is due to the source) is

$$
n(s, \hat{\mathbf{\Omega}})=q(r, \hat{\mathbf{\Omega}}) e^{-\Sigma s} .
$$


If the ray crosses a surface $S$ a distance $t$ from the source point along the ray, then the uncollided density on the surface due to a source at $r$ along the ray $\hat{\Omega}$ is

$$
n_{q}(S, \hat{\mathbf{\Omega}})=q(r, \hat{\mathbf{\Omega}}) e^{-\Sigma_{r}}
$$

Likewise, if the uncollided particle density crossing a surface at a point $r$ in direction $\hat{\Omega}$ into the cell with cross section $\Sigma$ is $n_{0}(r, \hat{\mathbf{\Omega}}$ ), then the uncollided density at the next surface crossing (on surface $S$ ) due to uncollided particles crossing the first surface at $r$ along the ray $\hat{\Omega}$ is

$$
n_{S}(S, \hat{\mathbf{\Omega}})=n_{0}(r, \hat{\mathbf{\Omega}}) e^{-\Sigma t}
$$

if the next surface crossing is a distance $t$ from point $r$.

In Monte Carlo parlance, $q$ and $n_{0}$ correspond to the particle source weight and the uncollided weight crossing a surface. In either case, the uncollided weight crossing the next surface, $w$, should be the source weight or surface-crossing weight $w_{0}$ (equal to $q$ or $n_{0}$ ) multiplied by the attenuation along the track length, $e^{-\Sigma t}$ :

$$
w=w_{0} e^{-\Sigma i} \text {. }
$$

The contribution of the track to a track-length uncollided flux tally in the cell is found by integrating Eq. (1), and its analog for the surface-crossing density, along the entire track, from $s=0$ to $s=t$. Again using $w_{0}$ to represent the source density or uncollided surface-crossing density, as appropriate, the uncollided density integrated along the track is

$$
n_{V}=w_{0} \int_{0}^{l} d s e^{-\Sigma s},
$$

which evaluates to

$$
n_{\nu}=\frac{w_{0}}{\Sigma}\left(1-e^{-\Sigma \imath}\right)
$$

when $\Sigma \neq 0$ and

$$
n_{v}=w_{0} t
$$

when $\Sigma=0$.

This simple analysis makes it clear how to compute uncollided surface or cell tallies in a Monte Carlo code. Start particles but do not let them have collisions. Instead, they travel from source to surface, then surface to surface, and at each surface their weight decreases by the attenuation along the distance they have traveled since the last surface [Eq. (4)]. This weight adjustment is sufficient for surface flux and current tallies. For cell flux tallies, score the weight entering times the distance along the track, as normal, if the cell is a void [Eq. (7)]; otherwise, score the weight entering multiplied by $\left(1-e^{-\Sigma t}\right) / \Sigma[$ Eq. (6)].

This strategy has been implemented in the MCNP6 subroutine hstory. The track length is always set to the distance to the next surface, dIs. Before the call to tal Iy for cell tallies, if $\Sigma \neq 0$, the track length is set to $\left(1-e^{-\Sigma t}\right) / \Sigma$; it is set back to $\mathrm{dls}$ on returning. Before processing the particle through the next surface, the weight is attenuated according to Eq. (4).

Note that pseudoparticles are still created and tracked as usual for any point detectors.

\section{User Interface and Documentation}

The user interface for the new NOTRN functionality is the same as for the old except that a fatal error has been added if a pulse-height (F8) tally or a mesh tally is requested (except MCNPX type 4 mesh tallies).

The documentation has to change only slightly. NOTRN is discussed in Vol. II of the manual, Chap. 3, Sec. IV.H. The "card type" there, in Table 3.11, and in Appendix A should be "Direct-Only Neutral Particle Tally Contributions." The paragraph describing the NOTRN card should be:

"When the NOTRN card appears in the INP file, no transport (scattering) of the neutral source particles takes place. Only the direct neutral particle source contributions to all point detector, surface, and cell tallies are made. This card is especially useful for doing a faster calculation to generate the direct tally. NOTRN is not legal in a continue run, and neither pulse-height tallies nor mesh tallies (except MCNPX type 4) are allowed with NOTRN." 


\section{Verification}

The uncollided surface and cell tally patch was tested using computational test objects with two materials, high-enriched uranium (HEU) and stainless steel (SS) 304. The density and composition of these materials are given in Table I. For the HEU density and composition given in Table I, the volumetric production rate ${ }^{6}$ of the four principal lines of uranium are given in Table II and the macroscopic cross sections ${ }^{7}$ for both materials are given in Table III. These cross sections do not include coherent scattering.

Table I. Material Specifications.

\begin{tabular}{c|c|c|c}
\hline Material & $\begin{array}{c}\text { Density } \\
\left(\mathrm{g} / \mathrm{cm}^{3}\right)\end{array}$ & Isotope & Wgt. Fraction \\
\hline HEU & 18.74 & $\mathrm{U}-235$ & 0.9473 \\
& & $\mathrm{U}-238$ & 0.0527 \\
\hline SS 304 & 7.86 & $\mathrm{Cr}-50$ & 0.008278 \\
& & Cr-52 & 0.159452 \\
& & Cr-53 & 0.018078 \\
& & Cr-54 & 0.004491 \\
& & Mn-55 & 0.020000 \\
& & Fe-54 & 0.040944 \\
& & Fe-56 & 0.642158 \\
& & Fe-57 & 0.014838 \\
& & Fe-58 & 0.001960 \\
& & Ni-58 & 0.061136 \\
& & Ni-60 & 0.023546 \\
& & Ni-61 & 0.001024 \\
& & Ni-62 & 0.003260 \\
& & Ni-64 & 0.000835 \\
\hline
\end{tabular}

Table II. Gamma-Ray Line Source Rates.

\begin{tabular}{c|c}
\hline $\begin{array}{c}\text { Line Energy } \\
(\mathrm{keV})\end{array}$ & $\begin{array}{c}\text { Source Rate } \\
\left(\gamma / \mathrm{cm}^{3} \cdot \mathrm{s}\right)\end{array}$ \\
\hline 144 & $1.499584 \times 10^{5}$ \\
186 & $7.859513 \times 10^{5}$ \\
766 & $3.809993 \times 10^{1}$ \\
1001 & $1.007587 \times 10^{2}$ \\
\hline
\end{tabular}

Table III. Gamma-Ray Line Cross Sections.

\begin{tabular}{c|c|c}
\hline $\begin{array}{c}\text { Line Energy } \\
(\mathrm{keV})\end{array}$ & $\begin{array}{c}\text { HEU } \\
(/ \mathrm{cm})\end{array}$ & $\begin{array}{c}\text { SS } \\
(/ \mathrm{cm})\end{array}$ \\
\hline 144 & $5.17590 \times 10^{1}$ & $1.46401 \times 10^{0}$ \\
186 & $2.76272 \times 10^{1}$ & $1.12674 \times 10^{0}$ \\
766 & $1.91450 \times 10^{0}$ & $5.31342 \times 10^{-1}$ \\
1001 & $1.42459 \times 10^{0}$ & $4.66985 \times 10^{-1}$ \\
\hline
\end{tabular}

The first test problem involves an isotropic point source of strength $q$ irradiating a disk of radius $R$ centered a distance $z$ from the source in a purely absorbing material with macroscopic cross section $\Sigma$. The total flux on the surface of the disk is ${ }^{1,8}$

$$
\begin{aligned}
\phi_{S}(z) & =\int_{\mu_{\min }}^{1} d \mu \psi(z, \mu) \\
& =\frac{q}{2} \int_{\mu_{\min }}^{1} d \mu \frac{e^{-\Sigma z / \mu}}{\mu} \\
& =\frac{q}{2}\left[E_{1}\left(\frac{\Sigma z}{\mu}\right)\right]_{\mu_{\min }}^{1} \\
& =\frac{q}{2}\left[E_{1}(\Sigma z)-E_{\mathrm{t}}\left(\frac{\Sigma z}{\mu_{\min }}\right)\right],
\end{aligned}
$$

where $\psi(z, \mu)$ is the angular flux on the disk and

$$
\mu_{\text {min }}=\left[(R / z)^{2}+1\right]^{\frac{1}{2}}
$$

is the minimum cosine (maximum angle) needed to subtend the disk from the source. The total current crossing the disk is 


$$
\begin{aligned}
J_{S}(z) & =\int_{\mu_{\min }}^{1} d \mu \mu \psi(z, \mu) \\
& =\frac{q}{2} \int_{\mu_{\min }}^{1} d \mu e^{-\Sigma z / \mu} \\
& =\frac{q}{2}\left[\mu e^{-\Sigma z / \mu}-\Sigma z E_{1}\left(\frac{\Sigma z}{\mu}\right)\right]_{\mu_{\text {min }}}^{1} \\
& =\frac{q}{2}\left(e^{-\Sigma z}-\mu_{\min } e^{-\Sigma z / \mu_{\min }}\right)-\frac{\Sigma z}{2}\left[E_{1}\left(\sum z\right)-E_{1}\left(\frac{\sum z}{\mu_{\min }}\right)\right] \\
& =\frac{q}{2}\left(e^{-\Sigma z}-\mu_{\min } e^{-\Sigma z / \mu_{\min }}\right)-\Sigma z \phi_{S}(z),
\end{aligned}
$$

where $\phi_{S}(z)$ is given by Eq. (8). The total flux in the volume between the source and the disk is

$$
\begin{aligned}
\phi_{V}(z) & =\int_{0}^{z} d z^{\prime} \phi_{S}(z) \\
& =\frac{q}{2} \int_{\mu_{\min }}^{1} d \mu \int_{0}^{z} d z^{\prime} \frac{e^{-\Sigma z^{\prime} / \mu}}{\mu} \\
& =\frac{q}{2} \int_{\mu_{\min }}^{1} d \mu\left(-\frac{\mu}{\Sigma}\right) \frac{1}{\mu}\left[e^{-\Sigma z^{\prime} / \mu}\right]_{0}^{z} \\
& =\frac{q}{2 \Sigma} \int_{\mu_{\min }}^{1} d \mu\left(1-e^{-\Sigma z / \mu}\right) \\
& =\frac{q}{2 \Sigma}\left(1-\mu_{\min }\right)-\frac{1}{\Sigma} J_{S}(z),
\end{aligned}
$$

where $J_{S}(z)$ is given by Eq. (10). Note that the derivation of Eq. (11) takes advantage of the fact that $\mu_{\min }$ is a constant for all of the disks in the volume between 0 and $z$.

The point source is $5 \mathrm{~cm}$ from the disk, which has a radius of $10 \mathrm{~cm}$, and emits the gamma-ray line energies of Table II, but each with a strength of $1 \mathrm{~s}^{-1}$. The material between the point source and the disk is SS 304. Analytic values for the surface flux, surface current, and volume flux are compared with MCNP values using the NOTRN capability and values using the FT special tally treatment in Table IV, Table V, and Table VI, respectively. The two MCNP calculations used $4 \times 10^{8}$ source photons. Both the NOTRN and the FT capabilities match the analytic results, but the NOTRN capability does so with uncertainties that are 4 to 58 times smaller for the surface tallies and 1.5 times smaller for the volume tallies. The NOTRN calculation was also faster by a factor of 2.5 .

Table IV. Surface Flux for Disk Problem

\begin{tabular}{c|r|r|r|r}
\hline & \multicolumn{1}{|c|}{$144 \mathrm{keV}$} & \multicolumn{1}{c}{$186 \mathrm{keV}$} & \multicolumn{1}{c}{$766 \mathrm{keV}$} & \multicolumn{1}{c}{$1001 \mathrm{keV}$} \\
\hline Analytic & $4.02733 \mathrm{E}-05$ & $2.74382 \mathrm{E}-04$ & $9.95325 \mathrm{E}-03$ & $1.50654 \mathrm{E}-02$ \\
& & & & \\
NOTRN & $4.02709 \mathrm{E}-05$ & $2.74433 \mathrm{E}-04$ & $9.95492 \mathrm{E}-03$ & $1.50652 \mathrm{E}-02$ \\
Uncertainty & $0.0152 \%$ & $0.0135 \%$ & $0.0102 \%$ & $0.0098 \%$ \\
Error $(\%)$ & $-0.0058 \%$ & $0.0187 \%$ & $0.0168 \%$ & $-0.0014 \%$ \\
Error $(N \sigma)$ & 0.385 & 1.384 & 1.644 & 0.145 \\
& & & & \\
FT & $3.97138 \mathrm{E}-05$ & $2.74470 \mathrm{E}-04$ & $9.95202 \mathrm{E}-03$ & $1.50688 \mathrm{E}-02$ \\
Uncertainty & $0.8850 \%$ & $0.3412 \%$ & $0.0593 \%$ & $0.0486 \%$ \\
Error $(\%)$ & $-1.3891 \%$ & $0.0322 \%$ & $-0.0124 \%$ & $0.0225 \%$ \\
Error $(N \sigma)$ & 1.592 & 0.094 & 0.208 & 0.463 \\
\hline \multicolumn{5}{c}{}
\end{tabular}


Table V. Surface Current for Disk Problem.

\begin{tabular}{c|r|r|r|r}
\hline & \multicolumn{1}{|c|}{$144 \mathrm{keV}$} & \multicolumn{1}{c|}{$186 \mathrm{keV}$} & \multicolumn{1}{c}{$766 \mathrm{keV}$} & \multicolumn{1}{c}{$1001 \mathrm{keV}$} \\
\hline Analytic & $3.62375 \mathrm{E}-05$ & $2.41091 \mathrm{E}-04$ & $8.05823 \mathrm{E}-03$ & $1.20245 \mathrm{E}-02$ \\
& & & & \\
NOTRN & $3.62350 \mathrm{E}-05$ & $2.41137 \mathrm{E}-04$ & $8.05981 \mathrm{E}-03$ & $1.20242 \mathrm{E}-02$ \\
Uncertainty & $0.0159 \%$ & $0.0144 \%$ & $0.0111 \%$ & $0.0107 \%$ \\
Error $(\%)$ & $-0.0069 \%$ & $0.0192 \%$ & $0.0197 \%$ & $-0.0023 \%$ \\
Error $(N \sigma)$ & 0.433 & 1.338 & 1.774 & 0.211 \\
& & & & \\
FT & $3.56888 \mathrm{E}-05$ & $2.41035 \mathrm{E}-04$ & $8.05926 \mathrm{E}-03$ & $1.20279 \mathrm{E}-02$ \\
Uncertainty & $0.8800 \%$ & $0.3386 \%$ & $0.0583 \%$ & $0.0477 \%$ \\
Error $(\%)$ & $-1.5142 \%$ & $-0.0231 \%$ & $0.0128 \%$ & $0.0285 \%$ \\
Error $(N \sigma)$ & 1.747 & 0.068 & 0.220 & 0.598 \\
\hline
\end{tabular}

Table VI. Volume Flux for Disk Problem.

\begin{tabular}{c|r|r|r|r}
\hline & \multicolumn{1}{|c|}{$144 \mathrm{keV}$} & \multicolumn{1}{c|}{$186 \mathrm{keV}$} & \multicolumn{1}{c}{$766 \mathrm{keV}$} & \multicolumn{1}{c}{$1001 \mathrm{keV}$} \\
\hline Analytic & $1.88767 \mathrm{E}-01$ & $2.45089 \mathrm{E}-01$ & $5.05013 \mathrm{E}-01$ & $5.66118 \mathrm{E}-01$ \\
& & & & \\
NOTRN & $1.88758 \mathrm{E}-01$ & $2.45104 \mathrm{E}-01$ & $5.05041 \mathrm{E}-01$ & $5.66135 \mathrm{E}-01$ \\
Uncertainty & $0.0087 \%$ & $0.0087 \%$ & $0.0087 \%$ & $0.0087 \%$ \\
Error $(\%)$ & $-0.0046 \%$ & $0.0062 \%$ & $0.0054 \%$ & $0.0029 \%$ \\
Error $(N \sigma)$ & 0.527 & 0.715 & 0.629 & 0.337 \\
& & & & \\
FT & $1.88772 \mathrm{E}-01$ & $2.45123 \mathrm{E}-01$ & $5.04952 \mathrm{E}-01$ & $5.66186 \mathrm{E}-01$ \\
Uncertainty & $0.0132 \%$ & $0.0132 \%$ & $0.0127 \%$ & $0.0125 \%$ \\
Error $(\%)$ & $0.0029 \%$ & $0.0139 \%$ & $-0.0122 \%$ & $0.0119 \%$ \\
Error $(N \sigma)$ & 0.216 & 1.057 & 0.961 & 0.954 \\
\hline \multicolumn{5}{r}{}
\end{tabular}

The second problem is a 10-kg sphere of HEU (radius of $5.03169067346416 \mathrm{~cm}$ ) enclosed tightly in an SS 304 shell of outer radius $7 \mathrm{~cm}$. Reference values of the total flux in the HEU and SS, the current flowing from the HEU into the SS, and the current leaking from the system were computed with the PARTISN multigroup discrete-ordinates transport code? with $S_{64}$ quadrature. These are compared with MCNP values using the NOTRN capability and values using the FT special tally treatment in Table VII, Table VIII, Table IX, and Table X, respectively, where differences are reported with respect to the PARTISN results. The two MCNP calculations used $4 \times 10^{8}$ source photons. Both the NOTRN and the FT capabilities match the PARTISN results, but the NOTRN capability does so with uncertainties that are 1.4 to 7 times smaller for the surface tallies and 1.5 to 2 times smaller for the volume tallies. The NOTRN calculation was also a factor of 1.6 faster. This problem verifies that the method of Sec. II works even when the tally cell is a source cell.

Table VII. Volume Flux in HEU for Sphere Problem.

\begin{tabular}{c|r|r|r|r}
\hline & \multicolumn{1}{|c|}{$144 \mathrm{keV}$} & \multicolumn{1}{c}{$186 \mathrm{keV}$} & \multicolumn{1}{c}{$766 \mathrm{keV}$} & \multicolumn{1}{c}{$1001 \mathrm{keV}$} \\
\hline PARTISN & $1.54157 \mathrm{E}+06$ & $1.50987 \mathrm{E}+07$ & $9.79705 \mathrm{E}+03$ & $3.38313 \mathrm{E}+04$ \\
& & & & \\
NOTRN & $1.54170 \mathrm{E}+06$ & $1.50991 \mathrm{E}+07$ & $9.79697 \mathrm{E}+03$ & $3.38290 \mathrm{E}+04$ \\
Uncertainty & $0.0087 \%$ & $0.0087 \%$ & $0.0089 \%$ & $0.0090 \%$ \\
Diff. (\%) & $0.0084 \%$ & $0.0026 \%$ & $-0.0008 \%$ & $-0.0068 \%$ \\
Diff. $(N \sigma)$ & 0.973 & 0.305 & 0.092 & 0.759 \\
& & & & \\
FT & $1.54183 \mathrm{E}+06$ & $1.50990 \mathrm{E}+07$ & $9.79673 \mathrm{E}+03$ & $3.38274 \mathrm{E}+04$ \\
Uncertainty & $0.0132 \%$ & $0.0132 \%$ & $0.0132 \%$ & $0.0131 \%$ \\
Diff. $(\%)$ & $0.0169 \%$ & $0.0020 \%$ & $-0.0033 \%$ & $-0.0115 \%$ \\
Diff. $(N \sigma)$ & 1.275 & 0.150 & 0.248 & 0.877 \\
\hline
\end{tabular}


Table VIII. Volume Flux in SS for Sphere Problem.

\begin{tabular}{c|r|r|r|r}
\hline & \multicolumn{1}{|c|}{$144 \mathrm{keV}$} & \multicolumn{1}{c}{$186 \mathrm{keV}$} & \multicolumn{1}{c}{$766 \mathrm{keV}$} & \multicolumn{1}{c}{$1001 \mathrm{keV}$} \\
\hline PARTISN & $1.53114 \mathrm{E}+05$ & $1.88695 \mathrm{E}+06$ & $2.20061 \mathrm{E}+03$ & $8.31826 \mathrm{E}+03$ \\
& & & & \\
NOTRN & $1.52935 \mathrm{E}+05$ & $1.88541 \mathrm{E}+06$ & $2.20051 \mathrm{E}+03$ & $8.31472 \mathrm{E}+03$ \\
Uncertainty & $0.1316 \%$ & $0.0962 \%$ & $0.0251 \%$ & $0.0216 \%$ \\
Diff. (\%) & $-0.1169 \%$ & $-0.0816 \%$ & $-0.0045 \%$ & $-0.0426 \%$ \\
Diff. $(N \sigma)$ & 0.889 & 0.849 & 0.181 & 1.968 \\
& & & & \\
FT & $1.53263 \mathrm{E}+05$ & $1.88285 \mathrm{E}+06$ & $2.20069 \mathrm{E}+03$ & $8.31229 \mathrm{E}+03$ \\
Uncertainty & $0.2540 \%$ & $0.1809 \%$ & $0.0432 \%$ & $0.0366 \%$ \\
Diff. $(\%)$ & $0.0973 \%$ & $-0.2173 \%$ & $0.0036 \%$ & $-0.0718 \%$ \\
Diff. $(N \sigma)$ & 0.383 & 1.204 & 0.084 & 1.960 \\
\hline
\end{tabular}

Table IX. HEU-to-SS Current for Sphere Problem.

\begin{tabular}{c|r|r|r|r}
\hline & \multicolumn{1}{|c|}{$144 \mathrm{keV}$} & \multicolumn{1}{c}{$186 \mathrm{keV}$} & \multicolumn{1}{c}{$766 \mathrm{keV}$} & \multicolumn{1}{c}{$1001 \mathrm{keV}$} \\
\hline PARTISN & $2.3047 \mathrm{E}+05$ & $2.2630 \mathrm{E}+06$ & $1.5744 \mathrm{E}+03$ & $5.5709 \mathrm{E}+03$ \\
& & & & \\
NOTRN & $2.30215 \mathrm{E}+05$ & $2.26119 \mathrm{E}+06$ & $1.57445 \mathrm{E}+03$ & $5.56882 \mathrm{E}+03$ \\
Uncertainty & $0.1316 \%$ & $0.0962 \%$ & $0.0250 \%$ & $0.0215 \%$ \\
Diff. (\%) & $-0.1106 \%$ & $-0.0800 \%$ & $0.0032 \%$ & $-0.0373 \%$ \\
Diff. $(N \sigma)$ & 0.842 & 0.832 & 0.127 & 1.739 \\
& & & & \\
FT & $2.30603 \mathrm{E}+05$ & $2.25998 \mathrm{E}+06$ & $1.57407 \mathrm{E}+03$ & $5.56779 \mathrm{E}+03$ \\
Uncertainty & $0.1862 \%$ & $0.1361 \%$ & $0.0356 \%$ & $0.0307 \%$ \\
Diff. $(\%)$ & $0.0577 \%$ & $-0.1335 \%$ & $-0.0210 \%$ & $-0.0558 \%$ \\
Diff. $(N \sigma)$ & 0.310 & 0.982 & 0.589 & 1.821 \\
\hline \multicolumn{4}{c}{}
\end{tabular}

Table X. Leakage Current for Sphere Problem.

\begin{tabular}{c|r|r|r|r}
\hline & \multicolumn{1}{|c|}{$144 \mathrm{keV}$} & \multicolumn{1}{c}{$186 \mathrm{keV}$} & \multicolumn{1}{c}{$766 \mathrm{keV}$} & \multicolumn{1}{c}{$1001 \mathrm{keV}$} \\
\hline PARTISN & $6.31466 \mathrm{E}+03$ & $1.36860 \mathrm{E}+05$ & $4.05075 \mathrm{E}+02$ & $1.68637 \mathrm{E}+03$ \\
& & & & \\
NOTRN & $6.31530 \mathrm{E}+03$ & $1.36813 \mathrm{E}+05$ & $4.05224 \mathrm{E}+02$ & $1.68597 \mathrm{E}+03$ \\
Uncertainty & $0.1527 \%$ & $0.1070 \%$ & $0.0258 \%$ & $0.0220 \%$ \\
Diff. (\%) & $0.0101 \%$ & $-0.0340 \%$ & $0.0369 \%$ & $-0.0240 \%$ \\
Diff. (No) & 0.066 & 0.318 & 1.429 & 1.088 \\
& & & & \\
FT & $6.36565 \mathrm{E}+03$ & $1.36761 \mathrm{E}+05$ & $4.05243 \mathrm{E}+02$ & $1.68451 \mathrm{E}+03$ \\
Uncertainty & $1.1212 \%$ & $0.5538 \%$ & $0.0707 \%$ & $0.0563 \%$ \\
Diff. $(\%)$ & $0.8074 \%$ & $-0.0720 \%$ & $0.0416 \%$ & $-0.1105 \%$ \\
Diff. $(N \sigma)$ & 0.714 & 0.130 & 0.588 & 1.966 \\
\hline
\end{tabular}

The spherical test problem was also done with a ring detector at a radius of $100 \mathrm{~cm}$. The reference result was obtained with a one-dimensional deterministic ray-tracing code. ${ }^{3}$ MCNP results were obtained with the NOTRN and FT capabilities, as before. Results are compared in Table XI. Clearly, the FT capability is not the best way to compute the uncollided point detector flux; after all, that is what the NOTRN capability is for! The results are almost exactly the same, however, and the NOTRN calculation was also a factor of 3.3 faster. This problem included all the surface and cell tallies presented above, demonstrating that uncollided surface and cell tallies do not interfere with uncollided point detector tallies. It was also verified that uncollided point detector fluxes are exactly the same when there are surface and cell tallies as when there are not. 
Table XI. Point Flux at $100 \mathrm{~cm}$ for Sphere Problem

\begin{tabular}{c|r|r|r|r}
\hline & \multicolumn{1}{|c|}{$144 \mathrm{keV}$} & \multicolumn{1}{c}{$186 \mathrm{keV}$} & \multicolumn{1}{c}{$766 \mathrm{keV}$} & \multicolumn{1}{c}{$1001 \mathrm{keV}$} \\
\hline Ray-trace & $5.03141 \mathrm{E}-02$ & $1.09023 \mathrm{E}+00$ & $3.22613 \mathrm{E}-03$ & $1.34306 \mathrm{E}-02$ \\
& & & & \\
NOTRN & $5.02543 \mathrm{E}-02$ & $1.09019 \mathrm{E}+00$ & $3.22509 \mathrm{E}-03$ & $1.34285 \mathrm{E}-02$ \\
Uncertainty & $0.1139 \%$ & $0.0805 \%$ & $0.0296 \%$ & $0.0181 \%$ \\
Diff. (\%) & $-0.1164 \%$ & $-0.0278 \%$ & $-0.0877 \%$ & $-0.0734 \%$ \\
Diff. $(N \sigma)$ & 1.023 & 0.346 & 2.969 & 4.065 \\
& & & & \\
FT & $5.02553 \mathrm{E}-02$ & $1.09019 \mathrm{E}+00$ & $3.22508 \mathrm{E}-03$ & $1.34285 \mathrm{E}-02$ \\
Uncertainty & $0.1139 \%$ & $0.0805 \%$ & $0.0296 \%$ & $0.0181 \%$ \\
Diff. $(\%)$ & $-0.1144 \%$ & $-0.0278 \%$ & $-0.0880 \%$ & $-0.0734 \%$ \\
Diff. $(N \sigma)$ & 1.005 & 0.346 & 2.978 & 4.064 \\
\hline \multicolumn{4}{c}{}
\end{tabular}

\section{Modifications}

Six source files were modified. Briefly, the files that were modified are:

newcrd.F90: issue a fatal error if not $n=1$ and there is an F8 tally.

itally.F90: issue a fatal error if not $r n=1$ and there is a mesh tally; if not $r n=1$ and any tally is type $1,2,4,6$, or 7 , reset notrn to 3 .

trnspt.F90: set nter $=$ al1_pars_loss_escape for notrn $=1$, not not $r \neq 0$.

hstory.F90: call expirs for zero or negative weight only if notrn $=0$; for notrn $=3$, set the track length to be the distance to the next surface, d ls; for not $n=3$, before making cell tallies, set the track length to be $\left(1-e^{-\Sigma t}\right) / \Sigma$, and after making cell tallies, reset the track length to $d s s$; for not $r n=3$, before processing through a cell boundary, accrue the attenuated weight to capture in the summary tables and reset the weight to wgt $=\mathrm{wgt} \times e^{-\Sigma t}$.

hstoryx.F90: same as hstrory.F90.

tallyd.F90: set $\operatorname{amfp}=$ ZERO for notr $n=1$, not notr $n \neq 0$.

The modified code passes the MCNP6 regression test set. The capability has been tested and works in continue runs. This capability is flagged internally with not $n=3$ instead of the more obvious not $r n=2$ so that it can be integrated with the LINES capability, ${ }^{4}$ which already uses not $r n=2$.

A complete listing of the Unix diffs between the modified subroutines and the original, unmodified subroutines from the initial release of MCNP6 follows. These changes can easily be made in earlier versions of MCNP.

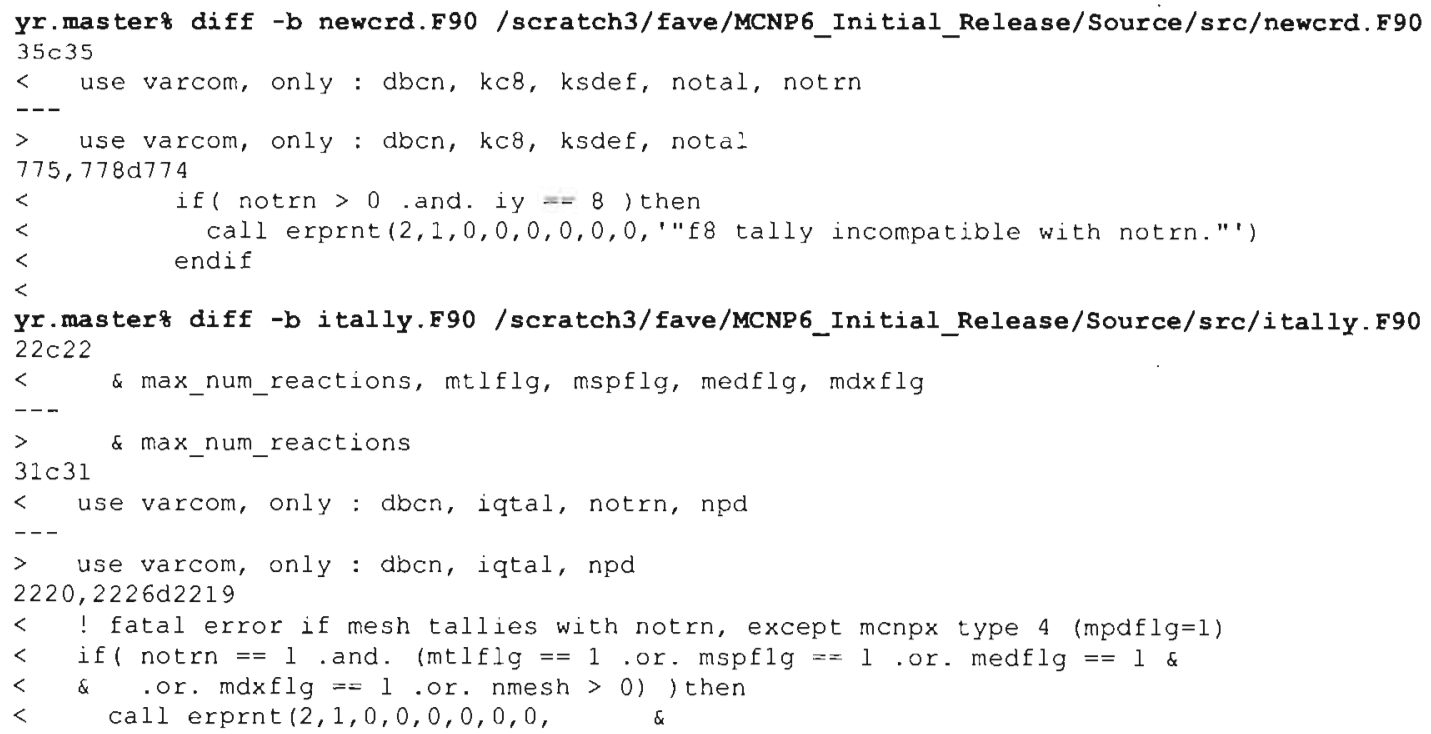




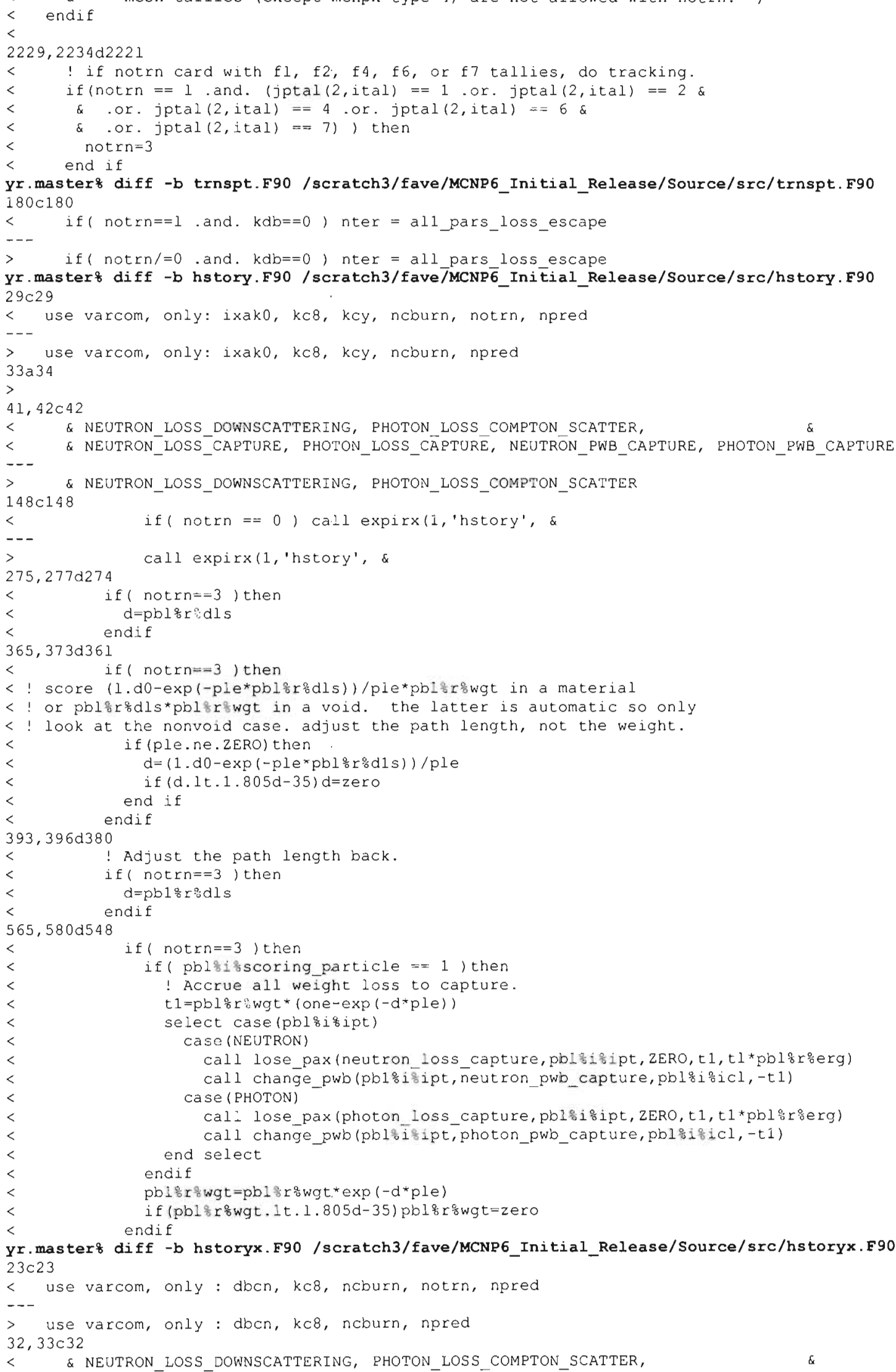




\section{Summary and Conclusions}

The MCNP "no transport" or NOTRN capability has been extended to allow uncollided flux and current tallies on surfaces and uncollided flux tallies in cells. The user interface is the same as in the standard capability. This capability is much more efficient than the "tally by collision" special tally treatment (FT INC) for computing uncollided quantities.

No other production Monte Carlo code that is generally available (KENO, MONACO, Mercury, MCBEND, and MONK were examined ${ }^{10-12}$ ) has this capability.

The capability should be extended further to work with mesh tallies.

The extended NOTRN capability has been integrated with the LINES capability (for surface and volume integrals of uncollided adjoint fluxes and forward-adjoint flux products) and the surface-flux tally enhancements ${ }^{8,13}$ in the MCNP6 initial release $^{5}$ (MCNP6_Betal). The thread version of the code with all of these features is MCNP6_IR_notrn2_5. 


\section{References}

1. Arthur B. Chilton, J. Kenneth Shultis, and Richard E. Faw, Principles of Radiation Shielding, Chap. 6, Prentice-Hall, Inc., Englewood Cliffs, New Jersey (1987).

2. X-5 Monte Carlo Team, "MCNP-A General Monte Carlo N-Particle Transport Code, Version 5," Vol. 11, LA-UR-030245, Los Alamos National Laboratory (rev. October 3, 2005).

3. Jeffrey A. Favorite, Keith C. Bledsoe, and David 1. Ketcheson, "Surface and Volume Integrals of Uncollided Adjoint Fluxes and Forward-Adjoint Flux Products," Nuclear Science and Engineering, 163, 1, $73-84$ (2009).

4. Jeffrey A. Favorite, "Surface and Volume Integrals of Uncollided Adjoint Fluxes and Forward-Adjoint Flux Products in Arbitrary Three-Dimensional Geometries Using MCNP," Transactions of the American Nuclear Society, 101, 633-635 (2009).

5. Tim Goorley, Mike James, Tom Booth, Forrest Brown, Jeff Bull, Larry Cox, Joe Durkee, Jay Elson, Mike Fensin, Art Forster, John Hendricks, Grady Hughes, Russ Johns, Brian Kiedrowski, Roger Martz, Stepan Mashnik, Gregg McKinney, Denise Pelowitz, Dick Prael, Jeremy Sweezy, Laurie Waters, Trevor Wilcox, and Tony Zukaitis, "MCNP6 Initial Release Notes: MCNP6_Beta 1," LA-UR-1 1-1765, Los Alamos National Laboratory (March 2011).

6. R. Gunnick and J. F. Tinney, "Analysis of Fuel Rods by Gamma-Ray Spectroscopy," Lawrence Livermore Laboratory report UCRL-51086 (1971), as quoted in R. H. Augustson and T. D. Reilly, "Fundamentals of Passive Nondestructive Assay of Fissionable Materials," Los Alamos Scientific Laboratory report LA-565I-M, Appendix A (1974).

7. Morgan C. White, "Photoatomic Data Library MCPLIB04: A New Photoatomic Library Based On Data from ENDF/B-VI Release 8," LA-UR-03-1019, Los Alamos National Laboratory (rev. February 7, 2003).

8. Jeffrey A. Favorite, Ashley D. Thomas, and Thomas E. Booth, "On the Accuracy of a Common Monte Carlo Surface Flux Grazing Approximation," Nuclear Science and Engineering, 168, 2, 115-127 (2011).

9. Ray E. Alcouffe, Randal S. Baker, Jon A. Dahl, Scott A. Turner, and Robert C. Ward, "PARTISN: A Time-Dependent, Parallel Neutral Particle Transport Code System," LA-UR-08-7258, Version 6.52, Los Alamos National Laboratory (November 2008). PARTISN version 6.52 was released in April 2011.

10. Douglas E. Peplow, Oak Ridge National Laboratory, private communication (October 19, 2011).

11. Scott McKinley, Lawrence Livermore National Laboratory, private communication (October 20, 2011).

12. Tim Fry, ANSWERS Software Service, private communication (October 20, 2011).

13. Jeffrey A. Favorite, "Monte Carlo Surface Flux Tallies," Proceedings of the International Conference on Mathematics and Computational Methods Applied to Nuclear Science and Engineering, Rio de Janeiro, Brazil, May 8-12, CD-ROM (2011). 


\section{JAF:jaf}

Distribution:

R. C. Little, XCP-DO, MS B259, rclelanl.gov

A. Sood, XCP-7, MS A 143, soodallanl,gov

D. A. Pimentel, HPC-1, MS F663, davidpelanl . gov

M. L. Fensin, D-5, MS K575, mfensinelanl.gov

J. S. Hendricks, D-5, MS K575, jxhelanl .gov

M. R. James, D-5, MS K575, mrjameselanl .gov

G. W. McKinney, D-5, MS K575, gwme l an l gov

F. B. Brown, XCP-3, MS A143, fbrownelanl.gov

J. S. Bull, XCP-3, MS A143, jsbullelanl.gov

R. A. Forster, XCP-3, MS A143, rafelan l.gov

J. T. Goorley, XCP-3, MS A143, jgoorleyelanl.gov

H. G. Hughes, XCP-3, MS A143, hghelanl.gov

B. C. Kiedrowski, XCP-3, MS A143, bckiedrodlanl.gov

G. P. Estes, XCP-7, MS F663, gpe@lanl.gov

J. P. Lestone, XCP-7, MS F663, lestonel lanl.gov

E. F. Shores, XCP-7, MS F663, eshores@lanl.gov

C. J. Solomon, XCP-7, MS F663, csolomone lanl.gov

B. A. Temple, XCP-7, MS F663, temple@lanl.gov

J. A. Favorite, XCP-7, MS F663, favedlanl.gov

X-Archive, xarchivedlanl.gov

XCP-DO File

XCP-7 File 